\title{
Pembrolizumab-induced autoimmune encephalitis in a patient with advanced non-small cell lung cancer: A case report
}

\author{
MAIKO NIKI, AYA NAKAYA, TAKAYASU KURATA, KAHORI NAKAHAMA, HIROSHIGE YOSHIOKA, \\ TOSHIHIKO KANEDA, KAYOKO KIBATA, MAKOTO OGATA and SHOSAKU NOMURA
}

First Department of Internal Medicine, Kansai Medical University, Hirakata, Osaka 573-1010, Japan

Received October 19, 2018; Accepted November 23, 2018

DOI: $10.3892 / \mathrm{mco} .2018 .1777$

\begin{abstract}
Immune checkpoint inhibitors have markedly changed lung cancer treatment and improved overall survival. However, immune checkpoint inhibitors may be associated with various adverse events, including encephalitis, although this complication is rare. We herein describe the clinical characteristics of a case of immune checkpoint inhibitor-induced encephalitis and its management. A 51-year-old man with squamous non-small cell lung cancer was receiving pembrolizumab treatment when he suddenly displayed an altered level of consciousness. Cerebrospinal fluid examination revealed elevated lymphocyte count and autoimmune encephalitis was suspected. The patient was promptly started on steroids and his consciousness immediately improved. Pembrolizumab treatment was discontinued; however, stable disease was maintained. In conclusion, encephalitis is a rare but possibly fatal adverse event of immune checkpoint inhibitors, and prompt diagnosis and treatment are mandatory.
\end{abstract}

\section{Introduction}

Although immune checkpoint inhibitors (ICPi) have markedly changed lung cancer treatment and improved overall survival (OS), they may be associated with immune-related adverse events (irAEs), including rash, pruritus, colitis, hepatitis, hypothyroidism and hypophysitis (1). Among ICPi-related irAEs, encephalitis is rare but it may be severe and potentially fatal. Its etiology remains elusive and diagnosis may be challenging, with only four cases of autoimmune encephalitis in patients with lung cancer treated with ICPi reported in the literature to date (2-4).

Correspondence to: Dr Aya Nakaya, First Department of Internal Medicine, Kansai Medical University, 2-5-1 Shin-machi, Hirakata, Osaka 573-1010, Japan

E-mail: nakaya1016@yahoo.co.jp

Key words: immune checkpoint inhibitor, non-small-cell lung cancer, pembrolizumab, autoimmune encephalitis, immune-related adverse event
We herein report the case of a patient undergoing pembrolizumab treatment for squamous non-small cell lung cancer (NSCLC) who developed autoimmune encephalitis, manifesting as altered level of consciousness, which immediately improved with steroid treatment.

\section{Case report}

A 51-year-old male patient was admitted to the Kansai Medical University Hospital (Osaka, Japan) in July 2016 with difficulty in walking and communicating. Magnetic resonance imaging (MRI) revealed a tumor in the right frontal lobe of the brain, which was subsequently resected and diagnosed as metastatic squamous cell carcinoma. The primary tumor was detected in his lung. The patient was diagnosed with squamous NSCLC, clinical stage IV, without epidermal growth factor receptor mutation or anaplastic lymphoma kinase translocation. On immunohistochemical staining, the lung specimen was $85 \%$ positive for programmed death ligand-1. The patient initially received whole-brain irradiation $(40 \mathrm{~Gy})$, followed by 4 cycles of chemotherapy (cisplatin and gemcitabine).

The patient achieved stable disease (SD), but the brain lesion grew in size after the chemotherapy; he then underwent irradiation and resection of the brain metastasis, followed by pembrolizumab as second-line treatment. Six months after initiation of pembrolizumab, the patient experienced a short seizure. A head MRI scan revealed no abnormality other than the previous surgical resection. Eight months after beginning pembrolizumab, the patient complained of fever, headache and unsteadiness in walking; however, no significant changes were detected on MRI. One week after this episode, the developed dizziness, difficulty in communication and altered level of consciousness. There was no muscle weakness, but was too unsteady to walk, and his conversation was scattered. On neurological examination, the patient had a Glasgow Coma Scale score of 10 (eyes opening to pressure, oriented, obeying commands, localizing pain), a negative Babinski sign and a positive Kernig sign; he also had a stiff neck. Head MRI showed no significant change compared with the previous MRI (Fig. 1A). Cerebrospinal fluid (CSF) examination revealed an elevated white blood cell (WBC) count $\left(58\right.$ cells $\left./ \mathrm{mm}^{3}\right)$ and total protein at $446 \mathrm{mg} / \mathrm{dl}$. Although CSF cytology revealed an elevated lymphocyte count (Fig. 1B), there was no evidence of infection. Electroencephalography revealed a slow wave in the right frontal 
robe, which indicated encephalitis or an aftermath of the previous brain metastasis. Autoimmune encephalitis was suspected, as the patient was under pembrolizumab treatment. The serum antibody tests for paraneoplastic neurological syndromes were all negative. Prednisolone $(2 \mathrm{mg} / \mathrm{kg}$ ) was initiated and the patient's level of consciousness immediately improved, although his walking remained unsteady for two weeks. Two weeks after beginning the prednisolone, the WBC count of the CSF decreased to 23 cells $/ \mathrm{mm}^{3}$ and the total protein to $57 \mathrm{mg} / \mathrm{dl}$. The prednisolone dose has been tapered to $10 \%$ per week, and the status of the lung cancer remains SD. The patient did not receive any other treatment after discontinuing pembrolizumab and the last follow up was performed in October 2018.

\section{Discussion}

Although ICPis have markedly changed lung cancer treatment, they may cause irAEs, including rash, pruritus, colitis, hepatitis, hypothyroidism and hypophysitis (1). Autoimmune encephalitis is a rare irAE, but it may be severe and potentially fatal. The etiology is unknown and diagnosis is challenging. Four cases of autoimmune encephalitis in patients with lung cancer treated with ICPi were found in the literature (2-4). Oligoclonal bands were found in 3 of these patients, although their clinical significance is unknown. One patient was positive for GAD65 antibody and another one was positive for an unclassified antibody; the remaining two patients were negative for any antibodies. Two patients received steroid pulse therapy and plasma exchange, but did not respond to treatment. Rituximab was then administered as a clinical trial, to which only one patient responded. The third patient responded to steroid pulse therapy, intravenous immunoglobulin pulse therapy and rituximab; and the fourth patient recovered with steroid pulse therapy alone.

The only definitive clinical characteristic for the diagnosis of autoimmune encephalitis is the presence of paraneoplastic antibodies. However, our patient was negative for all antibodies tested. CSF analysis revealed elevated WBC count and total protein level, with increased lymphocytes. As there was no evidence of infection, the recent administration of pembrolizumab was the only factor raising the suspicion of autoimmune encephalitis. The patient responded well to corticosteroid therapy, which confirmed autoimmune etiology.

It was previously reported that irAEs may affect nivolumab efficacy in NSCLC (5). Thus, the development of autoimmune encephalitis may reflect a strong response to pembrolizumab. In fact, our patient maintains SD without treatment following discontinuation of pembrolizumab.

Whether ICPis affect brain metastasis remains unclear $(6,7)$. Some prospective trials are currently being conducted, and the results of a phase 2 trial of pembrolizumab for melanoma and NSCLC with brain metastasis (8) indicate that the brain lesions were responsive in 6 of 18 patients with NSCLC. Trials of ipilimumab for melanoma with brain metastasis report intracranial response rates of $10-41.6 \%$ and median OS of 3.7-21.3 months $(9,10)$. These results indicate that ICPis may be of value as treatment for brain metastasis.

However, collective reports on irAE neurotoxicity are lacking, and rapid approval of ICPis may not adequately address their side effects. Furthermore, predicting irAEs is difficult, as they exhibit variable patterns, and the associated
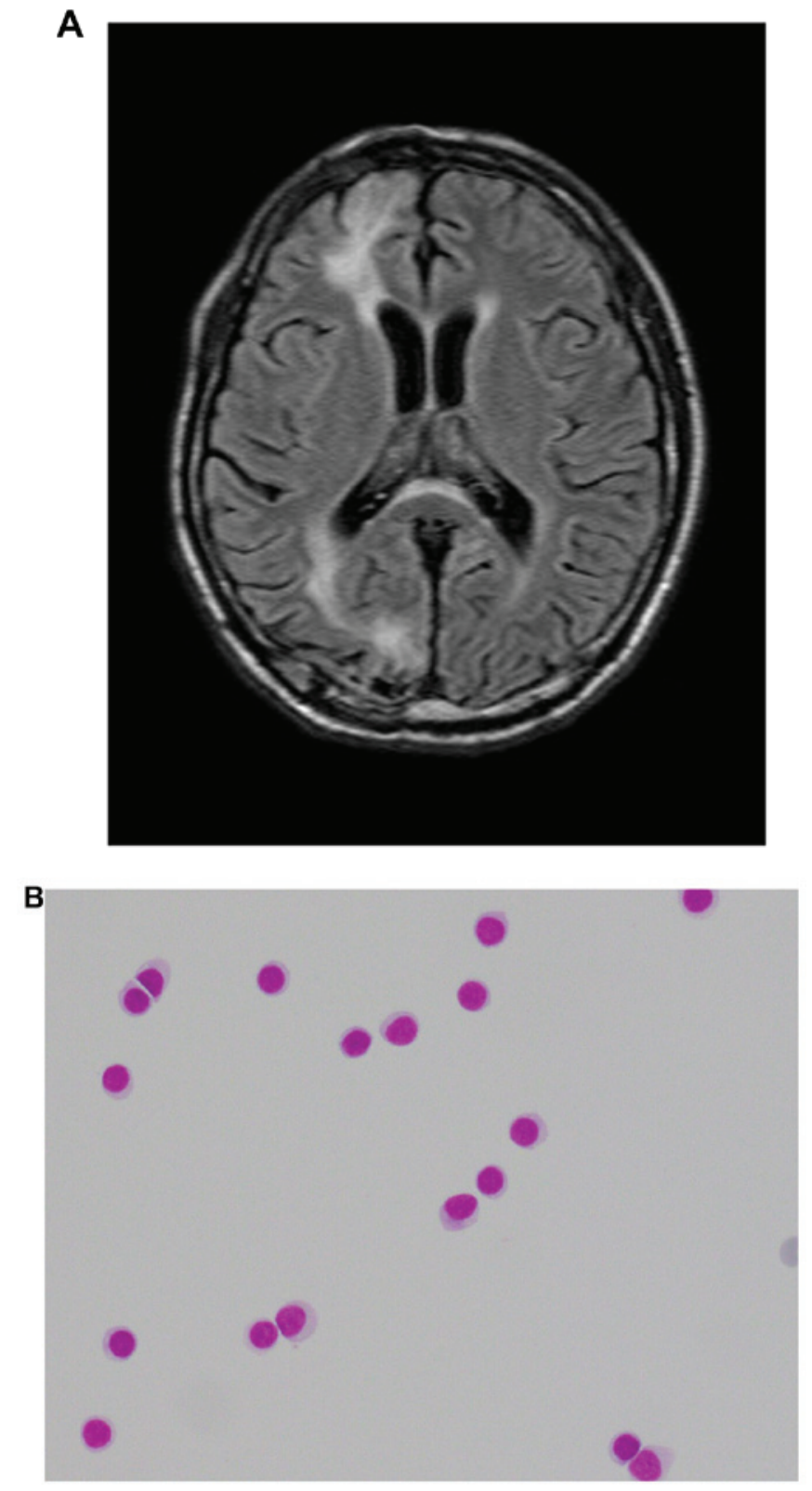

Figure 1. (A) Axial T1-weighted images demonstrated a prior surgical lesion without any new lesion indicating autoimmune encephalitis. (B) Cerebrospinal fluid cytology revealed an elevated lymphocyte count. Wright-Giemsa staining; magnification, x100.

factors have not been identified. Therefore, countermeasures against irAEs, particularly neurotoxicity, are urgently needed, and more relevant cases should be collected and studied.

\section{Acknowledgements}

Not applicable.

\section{Funding}

No funding was received.

\section{Ethics approval and consent to participate}

Not applicable. 


\section{Patient consent for publication}

The patient provided their informed consent for the publication of the case details and any associated images.

\section{Availability of data and materials}

Not applicable.

\section{Authors' contributions}

MN, AN, TaK, KN, HY, ToK, KK, MI and SN conceived and designed the current study, acquired the data and analyzed/interpreted the data. AN and TaK drafted the manuscript and revised it critically. AN, ToK and SN approved the manuscript for publication.

\section{Competing interests}

TaK received honoraria from MSD. The remaining authors have no potential conflicts of interest to disclose.

\section{References}

1. Weber JS, Kähler KC and Hauschild A: Management of immunerelated adverse events and kinetics of response with ipilimumab. J Clin Oncol 30: 2691-2697, 2012.

2. Schneider S, Potthast S, Komminoth P, Schwegler G and Böhm S: PD-1 Checkpoint inhibitor associated autoimmune encephalitis. Case Rep Oncol 10: 473-478, 2017.
3. Shah S, Dunn-Pirio A, Luedke M, Morgenlander J, Skeen M and Eckstein C: Nivolumab-induced autoimmune encephalitis in two patients with lung adenocarcinoma. Case Rep Neurol Med 2018: $1-4,2018$

4. Williams TJ, Benavides DR, Patrice KA, Dalmau JO, de Ávila AL, Le DT, Lipson EJ, Probasco JC and Mowry EM: Association of autoimmune encephalitis with combined immune checkpoint inhibitor treatment for metastatic cancer. JAMA Neurol 73: 928-933, 2016.

5. Nakaya A, Kurata T, Yoshioka H, Takeyasu Y, Niki M, Kibata K, Satsutani N, Ogata M, Miyara T and Nomura S: Neutrophil-tolymphocyte ratio as an early marker of outcomes in patients with advanced non-small-cell lung cancer treated with nivolumab. Int J Clin Oncol 23: 634-640, 2018.

6. O'Kane GM and Leighl NB: Are immune checkpoint blockade monoclonal antibodies active against CNS metastases from NSCLC?-current evidence and future perspectives. Transl Lung Cancer Res 5: 628-636, 2016.

7. Berghoff AS, Venur VA, Preusser M and Ahluwalia MS: Immune checkpoint inhibitors in brain metastases: From biology to treatment. Am Soc Clin Oncol Educ Book 36: e116-e122, 2018.

8. Goldberg SB, Gettinger SN, Mahajan A, Chiang AC, Herbst RS, Sznol M, Tsiouris AJ, Cohen J, Vortmeyer A, Jilaveanu L, et al: Pembrolizumab for patients with melanoma or non-small-cell lung cancer and untreated brain metastases: Early analysis of a non-randomised, open-label, phase 2 trial. Lancet Oncol 17: 976-983, 2016.

9. Larkin J, Chmielowski B, Lao CD, Hodi FS, Sharfman W, Weber J, Suijkerbuijk KPM, Azevedo S, Li H, Reshef D, et al: Neurologic serious adverse events associated with nivolumab plus ipilimumab or nivolumab alone in advanced melanoma, including a case series of encephalitis.Oncologist 22: 709-718, 2017.

10. Ahmed KA, Abuodeh YA, Echevarria MI, Arrington JA, Stallworth DG, Hogue C, Naghavi AO, Kim S, Kim Y, Patel BG, et al: Clinical outcomes of melanoma brain metastases treated with stereotactic radiosurgery and anti-PD-1 therapy, anti-CTLA-4 therapy, BRAF/MEK inhibitors, BRAF inhibitor, or conventional chemotherapy. Ann Oncol 27: 2288-2294, 2016. 